A N N A L E S Annales de Bretagne et des Pays de l'Ouest

\title{
Réfugiés kurdes de Turquie en Loire-Atlantique depuis les années 1970
}

Kurd refugees from Turkey in Loire-Atlantique: evolutions and trends since the 1970s

\section{Coline Rondeau}

\section{(2) OpenEdition}

\section{Journals}

Édition électronique

URL : http://journals.openedition.org/abpo/3728

DOI : $10.4000 /$ abpo.3728

ISBN : 978-2-7535-7411-3

ISSN : 2108-6443

Éditeur

Presses universitaires de Rennes

\section{Édition imprimée}

Date de publication : 20 décembre 2017

Pagination : $155-180$

ISBN : 978-2-7535-7409-0

ISSN : 0399-0826

\section{Référence électronique}

Coline Rondeau, «Réfugiés kurdes de Turquie en Loire-Atlantique depuis les années 1970 », Annales de Bretagne et des Pays de l'Ouest [En ligne], 124-4 | 2017, mis en ligne le 20 décembre 2019, consulté le 08 janvier 2020. URL : http://journals.openedition.org/abpo/3728 ; DOI : 10.4000/abpo.3728 


\title{
Réfugiés kurdes de Turquie en Loire-Atlantique depuis les années 1970
}

\author{
Coline RONDEAU \\ Doctorante en histoire contemporaine, université d'Angers, CERHIO CNRS FRE 2004
}

Islamisés entre les $\mathrm{VII}^{\mathrm{e}}$ et $\mathrm{XII}{ }^{\mathrm{e}}$ siècles, les Kurdes appartiennent au rameau iranien des peuples indo-européens. Cependant, ils sont identifiés en Turquie en tant que "Turcs" ou "Turcs des montagnes " car, depuis le traité de Lausanne (1923), seules les minorités religieuses (juifs, chrétiens) sont reconnues. Ainsi, les Kurdes ont vu leur appartenance ethnique niée au profit de leur appartenance religieuse. Sans État, certains Kurdes de Turquie ont fait le choix de s'exiler vers différentes terres d'asile comme la Suède, l'Allemagne, la France ${ }^{1}$.

Les premières vagues de migrations kurdes vers l'Europe se produisent dans les années 1960 grâce notamment à différents accords bilatéraux conclus avec la Turquie. Le gouvernement français signe, le 8 avril 1965, avec l'État turc, un accord de main-d'œuvre qui provoque l'arrivée d'une population masculine. Ce mouvement d'immigration s'intensifie à partir des années 1970 avec la venue des épouses et des enfants de ces travailleurs ${ }^{2}$. Le 3 juillet 1974, le gouvernement français suspend sa politique migratoire mais en 1976, les pouvoirs publics décident d'autoriser puis d'organiser l'immigration familiale ${ }^{3}$. Le 12 septembre 1980, la Turquie subit le coup d'État de Kenan Evren qui considérait le « nationalisme kurde comme [une] des pathologies à soigner ${ }^{4}$ ". Conséquemment, des villages kurdes sont détruits dans les régions de Siirt, Hakkari, Van, Sirnak... En réponse, le PKK

1. BozARSLAN, Hamit, La question kurde. États et minorités au Moyen-Orient, Paris, Presses de la Fondation Nationale des Sciences Politiques, 1997.

2. AKsAZ, Elif, L'émigration turque en France : 50 ans de travaux de recherche en France et en Turquie. Bibliographie commentée, proposée à l'occasion $d u 50^{e}$ anniversaire de la signature d'un accord bilatéral de main-d'œuvre entre la France et la Turquie le 8 avril 1965 , Istanbul, Istambul, IFEA, 2015.

3. LOCHAK, Danièle, « La politique d'immigration en France et l'évolution de la législation ", dans BRIBOSIA, Emmanuelle, REA, Andrea (dir.), Les nouvelles migrations. Un enjeu européen, Bruxelles, Complexe, 2002, p. 207-231.

4. Bozarslan, Hamit, Histoire de la Turquie. De l'Empire à nos jours, Paris, Tallandier, 2013, p. 368. 
(Partiya Karkerên Kurdistan) déclare, en août 1984, la lutte armée contre le gouvernement turc. Une décennie plus tard, en 1993, sous le gouvernement de Suleyman Demirel, ce sont les villages des régions de Bitlis, Diyarbakir, Mardin, Tunceli, Bingöl et Sivas qui sont détruits. En ruinant l'économie kurde cette méthode vise à l'anéantissement du mouvement kurde. En effet, la destruction des récoltes, le minage des champs empêchent la région du sud-est de se développer et obligent les habitants à migrer dans les villes de l'ouest : la ville de Diyarbakir devient la première ville kurde de Turquie. Plus que jamais, les Kurdes n'ont le choix qu'entre l'assimilation, la lutte ou le départ ${ }^{5}$.

En 1981, la gauche arrive au pouvoir en France; elle applique une politique d'ouverture en matière d'asile politique. Corinne Chevallier explique qu'à « la même époque, en Allemagne, où une forte communauté immigrée turque est implantée, le gouvernement mène une politique de dissuasion en matière de droit d'asile. En 1982, l'Allemagne détient le record de refus avec 81,1 \% [...]. En France, la même année, le taux moyen de décision de reconnaissance de la qualité de réfugié par l'OFPRA [Office Français de Protection des Réfugiés et des Apatrides] est de 73,88 \% ${ }^{6}$ ". Au cours de la décennie 1980, les demandeurs d'asile turcs et kurdes se tournent donc plutôt vers la France.

La représentation, consciente ou non, d'un " Ouest replié sur luimême ${ }^{7}$ ", d'une " terre de faible immigration " à l'intérieur du territoire français n'est pas conforme à la réalité puisque "la proportion de Turcs semble actuellement supérieure dans les Pays de la Loire à celle observée sur l'ensemble de la France ${ }^{8}$ ". En 1990, l'ouverture d'un consulat de Turquie à Nantes révèle la part importante des ressortissants turcs dans le département de Loire-Atlantique ${ }^{9}$. En parallèle, des chercheurs avancent qu'environ le tiers de la population turque émigrée en France est d'origine kurde ${ }^{10}$. Cependant, pour l'administration française, la nationalité prévaut sur l'origine ethnique. Puisqu'il n'existe pas de Kurdistan délimité par des frontières et reconnu par l'ensemble des

5. SCALBERT-YÜCEL, Clémence, « Le peuplement du Kurdistan bouleversé et complexifié : de l'assimilation à la colonisation ", L'information géographique, 2007, vol. 71, p. 63-86.

6. CHEVAlLIER, Corinne, "Itinéraire d'une communauté en exil : les réfugiés politiques turcs de gauche en France après le coup d'état militaire de septembre 1980 en Turquie ", thèse de doctorat en sociologie, EHESS, sous la direction de Hamit Bozarslan, 28 janvier 2010, p. 56.

7. DenÉChÈRE, Yves, MARAIS, Jean-Luc, "Introduction ", dans DenÉchÈRE, Yves, MARAIS, Jean-Luc (dir.), Les étrangers dans l'Ouest de la France (XVIII $-X X^{e}$ siècles), Annales de Bretagne et des pays de l'Ouest, tome $109, \mathrm{n}^{\circ} 4,2002, \mathrm{p} .7$.

8. Ponty, Janine, "L'Ouest de la France, terre atypique d'immigrations ", in DENÉCHÈRE, Yves, MARAIS, Jean-Luc (dir.), Les étrangers..., op. cit., p. 21.

9. Arch. dép. de Loire-Atlantique, $1944 \mathrm{~W} 133$, note des renseignements généraux, septembre 1992, n 72 , "Implantation des étrangers dans le département ", 5 p.

10. GÖKALP, Altan, "Conjoints et stratégies matrimoniales dans l'immigration ", Cahiers d'Études sur la Méditerranée Orientale et le monde Turco-iranien, 2006 [http://cemoti. revues.org/563]. 
États, les Kurdes sont identifiés comme "Turcs " ou "Turcs d'origine kurde ". Le peuple kurde est alors confondu avec le peuple turc, ce qui entraîne une invisibilité kurde en Turquie mais aussi en France. Les Turcs et les Kurdes appartiennent à deux contextes migratoires : migrants économiques et demandeurs d'asile/réfugiés. Le présent article vise à expliquer les stratégies migratoires mises en œuvre par ces hommes et ces femmes installés en Loire-Atlantique afin de comprendre comment et de quelle(s) manière(s) les Kurdes expriment ou dissimulent leur identité en fonction de ces différents contextes migratoires ${ }^{11}$.

Les sources utilisées pour cette recherche sont d'abord des documents de la Préfecture de Loire-Atlantique (cabinet du Préfet, bureau des étrangers, etc.) et de la Direction départementale des renseignements généraux conservés aux archives départementales de Loire-Atlantique ${ }^{12}$. Ces sources, consultées sur dérogation, sont constituées de rapports, notes de synthèse, statistiques, dossiers individuels d'étrangers au sein desquels sont conservées des cartes de résidence, de travail, des actes de mariage, des photocopies de passeport, des lettres écrites par les demandeurs d'asile adressées à l'OFPRA et/ou à la CRR (Commission des Recours des Réfugiés) ${ }^{13}$. Ces sources permettent de distinguer les différentes étapes dans l'acquisition de la qualité de réfugié et dans la demande de naturalisation. Elles révèlent des réseaux politiques ou familiaux tout en mettant en exergue des parcours individuels relatifs à plusieurs temporalités et contextes. Sur les 568 versements sélectionnés, 398 ont été consultés (soit $70 \%$ ) permettant de recenser 9 Kurdes et 649 Turcs (trois parcours de réfugiés kurdes seront développés : Z., M. et J.). Les noms, prénoms, lieux et dates de naissance, noms et prénoms des parents, adresses, dates de départ, nombre d'enfants, métiers, etc., ont été retranscrits dans une base de données. Celle-ci s'ajoute à une autre base de données (839 Turcs et 56 Kurdes) créée à partir

11. Cet article reprend des éléments de notre mémoire : RonDEAU, Coline, "Turcs et Kurdes de Turquie en Loire-Atlantique des années 1970 à nos jours. D'une analyse des stratégies migratoires à une réflexion sur l'identité kurde ", mémoire de master 2 en histoire, Université d'Angers, sous la direction de Yves Denéchère, 17 juin 2016.

12. Arch. dép. de Loire-Atlantique, 1138 W 37, Étrangers, logement : statistiques, correspondance, documentation, procès-verbaux de réunions (1970-1978); $1260 \mathrm{~W}$ 191, Étrangers, non admissions, immigration clandestine, admissions, refoulements, expulsions (1976-1985); 1286 W 2-17, 20-25, 54-75, 130-140, 159-171, 191-195, 219-224, 245-249, 255-300, étrangers : dossiers individuels (1946-1989); 1405 W 87, Associations et mouvements : rapports des renseignements généraux (1986-1987); 1505 W 155, Police - étrangers : documentation, statistiques, rapports des renseignements généraux. (1974-1986); $1680 \mathrm{~W}$ 1-56, Étrangers ayant acquis la nationalité française, étrangers sortis définitivement du territoire (1970-1997); 1683 W 1-25, Étrangers dont le titre de séjour est périmé (1970-1997); 1882 W 1-160, Étrangers ayant acquis la nationalité française (1965-2003); 1944 W 133, 135, 137, Chartes documentaires des renseignements généraux sur la situation de la vie politique en Loire-Atlantique, Mayenne, Vendée (1975-1995).

13. Pour plus d'informations, voir GRESLIER, Florence, "La Commission des Recours des réfugiés ou "l'intime conviction" face au recul du droit d'asile en France ", Revue européenne des migrations internationales, 2007, vol. 23, nº 2, p. 107-133. 
de sources consultées, sur dérogation, aux archives départementales de Maine-et-Loire ${ }^{14}$. Enfin, un dépouillement est en cours aux archives départementales d'Ille-et-Vilaine (251 Turcs et 32 Kurdes) ${ }^{15}$.

Ces sources écrites sont complétées par des sources orales indispensables pour une recherche d'histoire du temps présent. En Loire-Atlantique, depuis 2012, le centre culturel kurde (CCK) de Nantes vise l'objectif de regrouper les Kurdes lors de conférences et de manifestations diverses. Ce centre est organisé en dix commissions codirigées chacune par une femme et un homme : culture (musique, théâtre, danse), linguistique, diplomatie, relations internes, relations externes, etc. La commission des femmes du CCK de Nantes est autonome puisqu'elle est détachée du conseil d'administration : elle s'est affranchie fin 2015 sous le nom de "Zin 44 ». Ces femmes kurdes souhaitent défendre leur identité kurde et s'émanciper du patriarcat. Le CCK et Zin 44 ont constitué des relais essentiels pour construire un corpus de témoignages oraux. La personne contactée, membre du conseil d'administration du CCK, a été rencontrée à trois reprises depuis novembre 2015. De manière très claire, cet homme a manifesté sa méfiance car nous aurions pu faire partie des services secrets turcs ou être un(e) énième étudiant(e) voyeur(se) en quête de sensations. Cette appréhension est légitime car en parallèle de ce travail, le contexte géopolitique et la situation des Kurdes en Turquie et au Moyen-Orient se sont dégradés avec de nombreuses exactions commises à l'encontre des Kurdes de la part du gouvernement turc et des soutiens de Bachar El Assad. À ce contexte, se sont ajoutés, en janvier 2013, les assassinats de Sakîne Cansiz, Fidan Doğan et de Leyla Söleymez, dont l'auteur présumé est mort, en décembre 2016, avant la tenue de son procès ${ }^{16}$. Aussi, les échanges ont demandé du temps

14. Arch. dép. de Maine-et-Loire, 1018 W 61-62, 131, Instructions, correspondances, contrôles des étrangers (1962-1979); 1038 W 18-19, Recensements et statistiques (1977); 1038 W 21-22, Dossiers individuels de naturalisation (1975-1976); 1385 W 2-17, 26, 32-34, 38-42, Dossiers individuels de personnes naturalisées (1985-1992), statistiques semestrielles des titres de séjour délivrés (1985-1990), transmission à la DST de listes nominatives d'étrangers ayant demandé une carte de séjour [non daté], étrangers ayant quitté le Maine-et-Loire [non daté].

15. Dans le cadre de notre thèse sur les "Réfugiés kurdes de Turquie dans l'Ouest de la France (1980-2003) ", sous la direction de Yves Denéchère, des dépouillements sont en cours, dont aux Arch. dép. d'Ille-et-Vilaine, 1426 W 11-59, Naturalisations (1980-1994); 1427 W 2, 6, 11-12, 17, 52, 57, 95, 128, 130, 137, 158, 171, 183, 197, 200, 211, 214, 218, 233, Demandes de titres de séjours (1967-1994); 1429 W 50-51, Immigration de familles : dossiers de demandes (1980-1982); 1754 W 1-30, Échantillon de dossiers d'étrangers, de salariés et d'étudiants (1984-1994); 2752 W 1-41, Dossiers individuels de naturalisation ou de réintégration de la nationalité (1994-2001); 2770 W 1-29, 32-51, Naturalisations (1985-2005), regroupement familial, mesures d'éloignement, reconduite (1985-2008).

16. Pour plus d'informations sur cette affaire : [http://www.francetvinfo.fr/france/troisfemmes-kurdes-tuees-d-une-balle-dans-la-tete-a-paris_200775.html]; [http://www.france24. com/fr/20140117-turquie-france-assassinat-kurdes -paris-these-crime-etat]; [http://www. france24.com/fr/20161217-omer-gueney-assassin-trois-militantes-kurdes-pa ris-meurtavant-proces-pkk-sakine-cansiz]. 
avant qu'une certaine confiance s'installe et que des rencontres puissent s'organiser ${ }^{17}$.

Les sources écrites et orales permettent de reconstruire les parcours migratoires des demandeurs d'asile kurdes entre la Turquie et la France. Leurs migrations ont été comparées à celles des migrants économiques turcs installés en Loire-Atlantique à partir des années 1970. Les expériences vécues par les Kurdes doivent être reliées à celles des Turcs afin de comprendre les moments de ruptures et de connexions entre ces deux peuples. Ainsi se dégagent trois thématiques qui seront étudiées successivement : le contexte de départ de ces hommes et de ces femmes et les modalités de déplacement pour arriver en France, leurs installations et leurs circulations en Loire-Atlantique, l'affirmation ou non d'une identité kurde et des pratiques et moyens utilisés dans l'appropriation d'un nouveau territoire.

\section{Quitter la Turquie pour la France}

Dans leurs lettres adressées à l'OFPRA, les Kurdes racontent les exactions et les supplices qu'ils ont subis. Ceux-ci ont entraîné des réactions diverses de la part des Kurdes. Ils ont évalué les " enjeux " et les "risques " qu'il y avait à rester en Turquie. À partir d'un certain seuil qu'ils ont jugé critique et dangereux, ils ont fait le choix de partir pour la France. Leurs départs, les réseaux utilisés sont étudiés à partir des informations recueillies dans leurs dossiers respectifs et reliés aux migrations des Turcs.

\section{Le contexte turc : les « années de plomb»}

Le coup d'État du 12 septembre 1980 est décrit comme «beaucoup plus brutal et déstructurant que les précédentes interventions militaires ". En effet, " 81634 Kurdes auraient ainsi été arrêtés entre septembre 1980 et septembre 1982 et 1399 militants kurdistes (dont 640 militants du PKK) condamnés à des peines de prison ferme ${ }^{18}$ ". Qui plus est, "la prison militaire de Diyarbakir, à elle seule, abritait plus de 5000 personnes accusées d'avoir pris part aux activités du PKK ${ }^{19}$ ". La politique de Kenan Evren vise à restructurer les institutions étatiques à partir de l'enseignement des bases kémalistes ${ }^{20}$. Hamit Bozarslan explique que la Turquie kémaliste a "légitim[é] la nation comme musulmane, donc turque, intégr[é]

17. Suite aux échanges avec un membre du CKK, nous avons assisté au Newroz à Nantes (20 mars 2016). Le samedi 30 avril, nous avons participé à une journée dédiée aux femmes et organisée par Zin 44 au ccK. Au cours de cette journée, nous avons échangé avec deux femmes : la porte-parole de Zin 44 et une réfugiée kurde (B.) installée depuis 9 ans en France.

18. GrojeAn, Olivier, "La cause kurde de la Turquie vers l'Europe. Contribution à une sociologie de la transnationalisation des mobilisations ", thèse de doctorat en sociologie politique, EHESS, sous la direction de Hamit Bozarslan, mai 2008, p. 97.

19. YILMAZ, Özcan, La formation de la nation kurde en Turquie, Paris, PUF, 2013, p. 143.

20. Ces dernières appelées les " six flèches " adoptées en 1931 sont le nationalisme, le populisme, le réformisme, le républicanisme, l'étatisme et le laïcisme. 
par conséquent l'islamité comme condition d'appartenance à la turcité, justifi[é] la gestion directe du domaine religieux par l'État; supprim[é] donc toute autonomie interne de la sphère religieuse subordonnée, et fix[é] en même temps la laïcité comme l'objectif non négociable da la nation ${ }^{21}$ ". Le régime se nourrit alors des thèses développées par des intellectuels turcs au cours des années 1960, à l'image de l'historien Seyyid Ahmed Arvasi. La " synthèse turco-islamique " d'Arvasi, inspirée par le concept de " culture nationale " élaboré dans les années 1910 par le sociologue Ziya Gökalp, est adoptée par le régime.

En novembre 1983, le parti militaire MDP (Milliyetçi Demokrasi Partisi) perd les élections contre l'ANAP (Anavatan Partisi) et Turgut Özal devient premier ministre. Ce dernier "divise "la nation" en deux : un "pilier central", à savoir les classes moyennes, et des "exclus", comprenant, pêlemêle, la gauche radicale, les pauvres urbains et ruraux, les alévis, les Kurdes $^{22}$ ". Datée du 19 octobre 1983, la loi n 2932 interdit la langue kurde. En août 1984, Abdullah Öcalan (surnommé Apo) lance la guérilla rendant de ce fait le pays instable. L'intensification des opérations du PKK entraîne en juillet 1987 la mise en place d'un régime d'exception dans les villes kurdes. "Celles-ci, sur le plan de la sécurité, formaient une "région en état d'exception" dépendant d'un "gouverneur régional" doté des pouvoirs exceptionnels. Le gouvernement a également créé des forces armées spéciales et réorganisé les forces militaires présentes. Ces nouvelles mesures ont provoqué la mort de nombreux civils et des violations massives des droits de l'homme ${ }^{23}$. " Les élections de 1991 permettent à des députés " kurdistes " du SHP (Sosyal Demokrat Halkçı Parti) d'intégrer le parlement. Leur arrivée au parlement apparaît comme une reconnaissance progressive d'une réalité kurde, d'autant que la loi n 2932 est abrogée le 12 avril. Mais " à partir de 1993 [sous Suleyman Demirel], les escadrons de la mort sèment la terreur dans les régions kurdes, faisant, selon les estimations officielles, plus de 2000 victimes, politiciens, intellectuels, hommes d'affaires, souvent sans lien aucun avec le $\mathrm{PKK}^{24}$ ». L'histoire moderne des Kurdes est alors marquée selon l'historien anglais David McDowall par deux axes de réflexion : une opposition entre les Kurdes et les différents gouvernements qui souhaitent les contrôler et une lutte des Kurdes afin de se faire reconnaître comme une nation ${ }^{25}$.

\section{Lutter d'abord...}

"L'immigré n'existe pour la société qui le nomme comme tel qu'à partir du moment où il en franchit les frontières et en force le territoire; l'immi-

21. BozARSLAn, Hamit, "Islam, laïcité et la question de l'Empire Ottoman à la Turquie kémaliste ", Archives de sciences sociales des religions, janvier-mars 2004, no 125, p. 111.

22. BozArSlan, Hamit, Histoire de la Turquie..., op. cit., p. 372.

23. YILMAZ, Özcan, La formation de la nation kurde en Turquie..., op. cit., p. 144.

24. BozArSlan, Hamit, Histoire de la Turquie..., op. cit., p. 374.

25. Cité par YILmAZ, Özcan, La formation de la nation kurde en Turquie..., op. cit., p. 1. 
gré "naît" de ce jour à la société qui le désigne de la sorte. Aussi, celle-ci s'autorise-t-elle à tout ignorer de ce qui précède en ce moment et cette naissance $^{26}$ ". La compréhension de la mobilité nécessite de s'intéresser à un " avant " et à un " après " pour les Kurdes. Ces derniers ne sont pas devenus Kurdes à leur arrivée en Loire-Atlantique. Leurs lettres adressées à l'OFPRA entre les années 1988 et 1998 montrent des degrés variés d'appartenance à l'identité kurde et à des formes de résistance. Ces sources écrites appellent l'historien(ne) à rendre compte des traumatismes vécus, à étudier la lexicologie et à faire attention au temps d'écriture : quand le réfugié est-il parti de Turquie? Quand écrit-il cette lettre?

Alors âgé de 25 ans, J. est arrivé en juillet 1989. Sa lettre semble avoir été écrite le jour même de son entrée en France. Il explique la turquisation progressive du pays par des déplacements forcés. Sa famille a été contrainte, au cours de la décennie 1960, de quitter Arsin (province de Trabzon) pour la province d'Erzincan. Il écrit que « le but c'est l'assimilation des Kurdes " et que " dans les écoles ils nous imposaient la politique turque $^{27}$ ". Les écoles sont considérées comme le vecteur essentiel pour éloigner les individus de leurs racines traditionnelles et religieuses. Dès les années 1950, le souci d'assimiler les Kurdes est une nécessité aux yeux du gouvernement turc. Dans des villages kurdes des écoles primaires sont créées, au sein desquelles les enseignants sont chargés de diffuser la langue et la culture turques. Le dessein de l'État-nation vise à l'instauration d'une langue commune (" théorie solaire de la langue ${ }^{28}$ ") et à l'homogénéisation du pays en prouvant la " continuité historique et ethnique en Anatolie ${ }^{29}$ ". Ainsi, l'éducation des jeunes turcs et non-turcs est axée sur une histoire commune dont l'objectif est d'exercer un contrôle moral permettant de réaliser l'idéal nationalitaire ${ }^{30}$. Mais la " scolarisation des Kurdes a abouti, d'une part, à la formation des élites [...], et d'autre part, à l'émergence d'un public attentif aux solutions proposées par ces élites, comme la création d'une nouvelle identité de groupe ${ }^{31}$ ". Issu d'une famille militante (son oncle et ses frères dont l'un est décédé), J. ne fut pas assimilé. Son engagement s'inscrit profondément dans la continuité familiale. Il détaille dix années de sa vie au cours desquelles ses rencontres familiales et extérieures ont

26. SAYAD, Abdelmalek, L'immigration ou les paradoxes de l'altérité, Bruxelles, De Boeck, 1991, p. 15-16.

27. Arch. dép. de Loire-Atlantique, 1882 W 138, lettre de J. au directeur de l'OFPRA, 16 juillet 1989, Nantes, p. 1.

28. "La théorie solaire de la langue " développée au cours de la décennie 1930 tentait de prouver l'origine turque de toutes les langues.

29. Bozarslan, Hamit, La question kurde. États et minorités..., op. cit., p. 77.

30. Le discours officiel tend à s'attribuer la " causalité première d'une série d'évènements : le passé, recomposé sur la base d'une uchronie, est présenté comme si un phénomène historique complexe n'avait pas un nombre infini de causes, mais une seule, l'intervention des Turcs dans l'histoire ", in COPEAUX, Étienne, Espaces et temps de la nation turque. Analyse d'une historiographie nationaliste, 1931-1993, Paris, CNRS Éditions, 1997, p. 42.

31. YILMAZ, Özcan, La formation de la nation kurde..., op. cit., p. 83. 
influencé et modifié son combat pour la cause kurde. Il dépeint ses déplacements forcés pour ne pas être arrêté par la police, ainsi que la mort de nombreux amis en donnant les noms, dates et circonstances du décès. Son vocabulaire est marqué par la pensée marxiste, accentuée par un champ lexical de la violence : " luttes ", " socialisme ", " classes ", " junte fasciste ", " oppressions ", " torture ", " tuerie ", " massacre ", " cadavres ".

Dans la lettre qu'il écrit une semaine après son arrivée en France en août 1998, M. indique avoir fait des études en Turquie et être allé jusqu'au lycée. Malgré la propagande turque au sein des écoles, il n'a pas été " endoctriné ", tout comme J. L'école est le premier lieu de résistance pour ces deux Kurdes. Par contre, M. a été influencé par " des personnes ayant des activités politiques plus ou moins légales " et a participé à la distribution de prospectus à l'origine de son arrestation ${ }^{32}$. Après sa détention, il a intégré le HADEP (Halkın Demokrasi Partisi). Il fut arrêté de nombreuses fois à cause de son militantisme pour mobiliser le peuple kurde. Le combat de cet homme de 28 ans, originaire d'Afsin (province de Kahramanmaraş), ne semble pas s'enraciner dans sa sphère familiale puisqu'il ne parle pas d'actions menées par celle-ci. Néanmoins, les relégations arbitraires de l'administration turque à l'encontre de son père, les licenciements dont M. a été l'objet en raison de son origine kurde et de ses activités politiques, ont été un terreau propice à son engagement politique.

La lettre de Z., jeune femme de 19 ans, écrite onze jours après son entrée en France en juillet 1993, témoigne de son engagement auprès des " mouvements séparatistes ". Cette expression laisse penser qu'il s'agit du PKK. Le soutien de Z $\mathrm{Z}$ à ces mouvements ne découle pas d'un univers familial engagé qui lui aurait transmis des valeurs militantes. Peut-être que cette transmission n'a pas été effective car Z. est une femme? Son engagement tire, sans doute, son origine de la propagande effectuée par les militants des partis et du PKK qui encouragent la mobilisation des Kurdes. Z. est une fille de paysans et sa coopération avec les mouvements séparatistes semble entraîner celle de ses parents. La propagande est essentielle compte tenu des exactions perpétrées par le gouvernement turc. Ce dernier a pour objectif d'épuiser psychologiquement et physiquement les villageois afin qu'ils cessent de soutenir matériellement les combattants du PKK. Ainsi sont pratiqués la destruction ou le pillage des récoltes, l'abattage des animaux, l'incendie des maisons obligeant les villageois à quitter leur village ${ }^{33}$. Cette violence étatique rompt les liens entre les villageois et les militants du PKK et provoque aussi une destruction économique du sud-est. La violence a transformé Z. Elle est la seule à évoquer clairement ses sentiments à l'encontre de l'État turc : " [L]es corps de ces hommes abattus comme

32. Arch. dép. de Loire-Atlantique, 1882 W 142, lettre de M. au directeur de l'OFPRA, 7 août 1998 , Nantes, p. 1.

33. Un réfugié kurde témoigne de l'incendie de sa maison par l'armée turque : Arch. dép. de Loire-Atlantique, 1882 W 38, lettre de Y. au directeur de l'OFPRA, 28 août 1994, Nantes, 1 p. 
des chiens. Un sentiment de haine m'a prise contre l'armée turque et ses commanditaires $[\ldots]^{34}$."

J. raconte avoir été choqué lors de l'assassinat de son frère. Cet élément est singulier puisqu'il n'exprime qu'une fois ce qu'il a pu ressentir. Par contre, tout au long de sa lettre, il insiste sur les noms de ses amis décédés et les conditions dans lesquelles ils sont morts. Son insistance révèle un traumatisme. Les évènements à répétition tels que les tortures, les menaces, la surveillance de la police, les fouilles, les gardes à vue... sont présents dans sa lettre et celle de $\mathrm{M}$. : " après le massacre de mes neuf amis ", " une procédure de 670 ans a été ouverte ", " après l'arrestation et la tuerie de [...] ", " mes 4 amis [...] ont été massacré[s] par la police ", " il a été emprisonné 10 ans ", "j'ai été mis en garde à vue le [...], j'ai été détenu pendant six mois ${ }^{35}$ ", etc.

Ces événements traumatiques démontrent l'impact de la violence sur les hommes, qu'elle soit prodiguée par l'armée turque ou par les guérillas kurdes. Les déplacements répétés des militants kurdes (Istanbul, Tunceli, Izmir...) sollicitent de nombreux réseaux. Leur errance choisie devient progressivement forcée pour fuir l'armée turque. Cette situation intenable sur le long terme, pour eux et pour leurs hôtes, les obligent à quitter la Turquie.

\section{...Puis se résigner à partir}

Z. a été prévenue de la menace d'une potentielle dénonciation à propos de son aide apportée au PKK. Elle a dû quitter Elbistan (province de Kahramanmaraş) et se rendre à Gaziantep (province de Gaziantep) chez l'un de ses parents ${ }^{36}$; elle ne mentionne ni le nom, ni le lien de parenté avec ce parent, sans doute pour le protéger. La notion de famille est très élargie pour les Kurdes et de nombreux " parents " ont été éparpillés sur tout le territoire à cause des déplacements provoqués par l'armée turque. La destruction des villages est un des facteurs déclencheurs des circulations kurdes au sein de l'espace turc. J. et Z. sont partis de la Turquie par Istanbul. Cette métropole, qui s'articule entre l'Europe et l'Asie, est un axe majeur au sein des migrations internationales dont l'importance a augmenté avec la fin du rideau de fer (1989) et la dislocation de l'URSS (1990-1991). Istanbul est " le comptoir, le hub, le sas et l'impasse " de la Turquie c'est-à-dire un "pôle d'interconnexions ${ }^{37}$ ".

34. Arch. dép. de Loire-Atlantique, 1683 W 1, lettre de Z. à l'OFPRA, 31 juillet 1993, Angers, p. 1-2.

35. Arch. dép. de Loire-Atlantique, 1882 W 138, lettre de J. au directeur de l'OFPRA 16 juillet 1989, Nantes, p. 1-2.; 1882 W 142, lettre de M. au directeur de l'OFPRA, 7 août 1998, Nantes, p. 1.

36. Arch. dép. de Loire-Atlantique, $1683 \mathrm{~W}$ 1, lettre de Z. à l'OFPRA, 31 juillet 1993, Angers, p. 2.

37. Aslan, Mustafa, Pérouse, Jean-François, « Istanbul : le comptoir, le hub, le sas et l'impasse. Fonctions dans le système migratoire international ", Revue européenne des migrations internationales, 2006, $\mathrm{n}^{\circ}$ 3, vol. 19, p. 173-204. 
Quant à M., il a quitté la Turquie par Izmir. Cette ville bénéficie d'une façade maritime sur la mer Égée et possède le deuxième plus grand port de Turquie après Istanbul. Par leur situation géographique, ces deux villes permettent aux migrants d'accéder à l'Europe par la Grèce, la Bulgarie.

Tous mentionnent la multiplicité des différents moyens de transport utilisés : camions (TIR : Transit International Routier), voitures (souvent des taxis), bateaux, trains, engendrant une diversité des routes empruntées et des durées de trajet variant entre une semaine et un mois et demi. Les indications sur le temps passé à voyager sont assez précises. On apprend la date de leur départ de Turquie et la date de leur arrivée en France. J. est un cas particulier puisqu'il mentionne toutes ses dates d'arrivée dans chaque ville. Cette précision pose question sur l'exactitude de ces informations. A-t-il été informé du temps nécessaire lors des préparatifs de ce voyage? A-t-il tenu un carnet de bord? Comment a-t-il su qu'il arriverait dans telle ville alors que ses compatriotes mentionnent (pour la plupart) des pays? Pourquoi tant de précision? On constate que l'axe privilégié (d'après les dossiers de Kurdes découverts aux archives départementales de Loire-Atlantique) est la route des Balkans qui permet d'accéder aux régions du nord de l'Italie (Frioul-Vénétie Julienne, Trentin Haut-Adige, Lombardie, Val d'Aoste, Piémont, Ligurie) supposant l'existence d'un trafic important de TIR reliant la Turquie à l'Italie. Comme le souligne Stéphane de Tapia : "Munich, Milan sont les deux principaux terminaux du trafic TIR turc, reliés par les ports de Venise et Trieste à ceux d'Istanbul, Izmir et Antalya, et par la route des Balkans, aujourd'hui détournée par l'Adriatique et la Hongrie, mais avec les mêmes terminaux $^{38}$. " Avant que n'ait lieu cette " déviation ", la route des Balkans s'ouvrait aux Turcs par la ville de Sofia en Bulgarie.

Sur la carte ci-jointe, les trajets de J. et M. sont présentés. J. est parti d'Istanbul dans un camion (TIR) qui l'a emmené jusqu'à Milan (Lombardie) en passant sûrement par la Bulgarie et les Balkans. De Milan, il est arrivé à Gênes en Ligurie (l'un des premiers ports italiens) afin d'accéder à San Remo, ville frontière avec la France. Il est entré par Nice puis Marseille, a rejoint la région parisienne puis a atteint Nantes. Son trajet a duré six jours ${ }^{39}$. M. est parti d'Izmir par bateau afin de rejoindre Marseille sept jours plus tard. Ses parents sont venus le chercher et l'ont emmené à Nantes ${ }^{40}$. Quant à Z., elle a reçu le soutien de ses parents qui ont contacté un chauffeur de TIR à Istanbul qui l'a conduite jusqu'en France (Angers en Maine-

38. TAPIA, Stéphane DE, "Échanges, transports et communications : circulation et champs migratoires turcs ", Revue européenne des migrations internationales, 1996, nº 2, vol. 12 , p. 52 .

39. Arch. dép. de Loire-Atlantique, 1882 W 138, lettre de J. au directeur de l'OFPRA, 16 juillet 1989, Nantes, p. 3.

40. Arch. dép. de Loire-Atlantique, 1882 W 142, lettre de M. au directeur de l'OFPRA, 7 août 1998, Nantes, p. 2. 
et-Loire). Ayant voyagé cachée pendant un mois et demi, elle ne cite pas les pays traversés ${ }^{41}$.

\section{Figure 1 - Parcours migratoires de M. et J. de la Turquie jusqu'à la France}

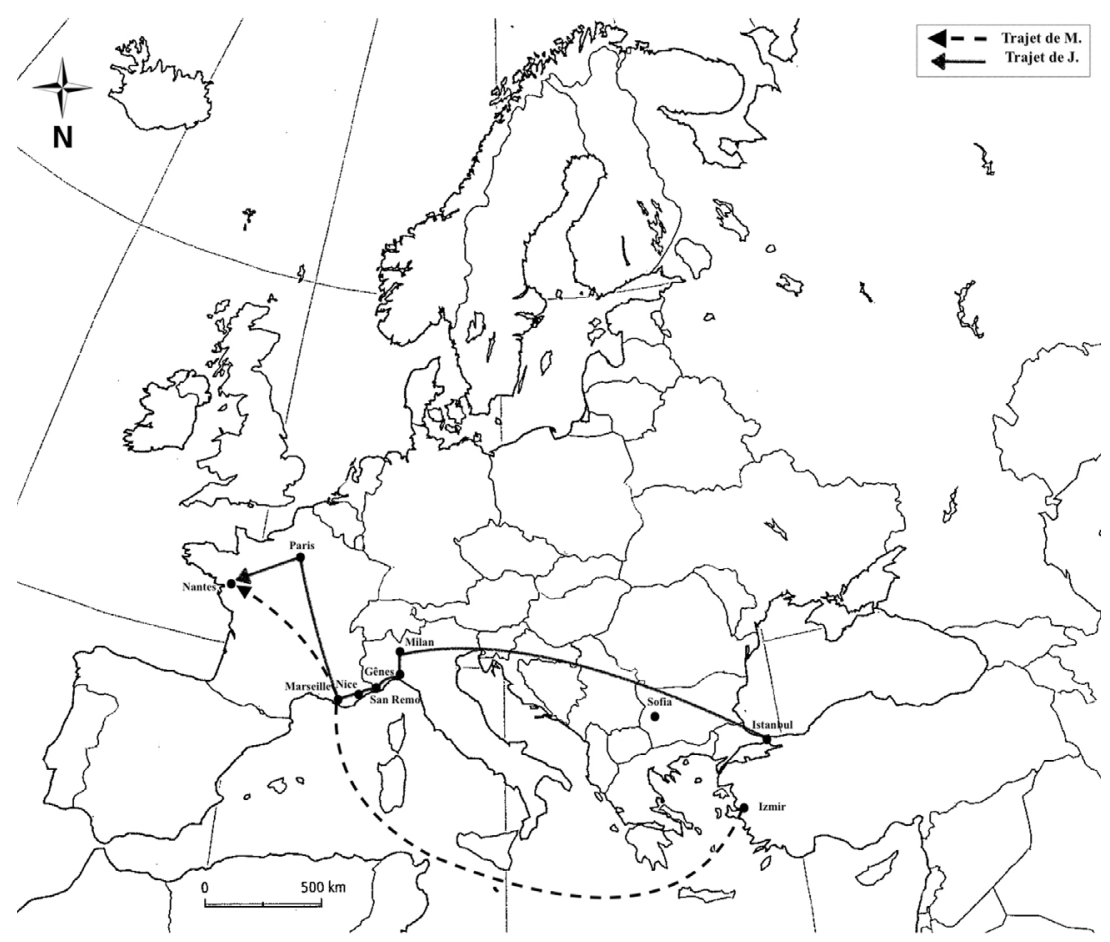

La capacité des migrants à se créer des relations est essentielle dans leur démarche migratoire. Ainsi, J. est entré en France muni d'un faux passeport accentuant encore plus l'importance d'un réseau mafieux ${ }^{42}$. Comme le souligne la géographe Chadia Arab, "le migrant saisit des occasions ${ }^{43}$ ". La construction d'un territoire circulatoire est le résultat de rencontres, d'échanges au cours desquels le migrant a la possibilité d'agir. Le migrant est un acteur actif. Leur appartenance à un groupe politique/syndical a

41. Arch. dép. de Loire-Atlantique, 1683 W 1, fiche d'information concernant un demandeur d'asile (Z.) par la préfecture de Loire-Atlantique, Nantes, [non datée], 1 p.

42. Arch. dép. de Loire-Atlantique, 1882 W 138, lettre de l'avocat de J. au président de la CRR, 25 juillet 1990, Paris, p. 4.

43. ARAB, Chadia, "Circulants marocains en réseau. La diversité des itinéraires et des routes migratoires ", in CORTÈs, Geneviève, FARET, Laurent, Les circulations transnationales. Lire les turbulences migratoires contemporaines, Paris, Armand Colin, 2009, p. 78. 
pu permettre à M. et J. d'utiliser des réseaux préexistants. M. est le seul à y faire allusion : "j'ai été aidé et renseigné par des personnes qui sont déjà allées en Europe pour des activités politiques. On m'a présenté une personne qui travaillait sur un navire. Moyennant 2000 dollars [... ${ }^{44}$." Ces propos témoignent de l'existence d'une filière clandestine sur laquelle s'appuieraient des militants politiques. M. ne nomme personne, ne donne ni le nom du navire sur lequel il a embarqué, ni des précisions sur le trajet effectué par celui-ci. La seule mention précise est celle de la somme déboursée. Il ne dit pas s'il s'agit de ses économies ou s'il a été aidé par des membres de sa famille (préfinancement de la traversée). Mais il est le seul à préciser un montant pour le trajet accompli. Ces échanges financiers ne peuvent être étudiés sans l'utilisation des archives de banques ou d'intermédiaires financiers. Dès lors, ces échanges ne sont pas perceptibles en termes de flux, de sommes engagées.

Les trois cas exposés témoignent de la difficulté à étudier les différents types de réseaux du fait de la diversité dans le contenu des lettres des Kurdes. L'attention est portée sur les conditions de vie en Turquie des trois migrants, sur les traumatismes qu'ils ont subis et non sur le montant payé pour quitter la Turquie. Ils ont été aidés par des réseaux sociaux que l'on peut qualifier de familiaux et par conséquent d'ethniques; tel est le cas pour $\mathrm{Z}$. Le poids de la famille transparaît le plus souvent au sein des sources consultées mais ne doit pas masquer celui des réseaux politiques et/ou syndicaux. Certains appartiennent à des familles politisées, militantes pouvant bénéficier de soutiens en dehors de la Turquie. Lorsque M. arrive à Marseille, ses parents sont présents pour l'emmener à Nantes. On peut alors prétendre que " c'est à l'intérieur d'un système familial multipolaire que la circulation trouve à la fois sa faisabilité et son sens " créant de "nouvelles territorialités ${ }^{45}$ ". Cette présence familiale atteste d'une continuité des réseaux tout au long du trajet du migrant ainsi que de l'emploi de moyens de télécommunication nécessaires pour la mise en place d'un tel projet migratoire. Leur utilisation articule une relation entre le local et le global dépassant les territoires au sein desquels les migrants circulent. Se dessine alors un champ migratoire, un " espace parcouru, pratiqué par les migrants " qui est "structuré par des flux importants, significatifs " englobant " le pays de départ et le pays d'emploi ${ }^{46}$ ".

44. Arch. dép. de Loire-Atlantique, 1882 W 142, lettre de M. au directeur de l'OFPRA, 7 août 1998, Nantes, p. 2.

45. SIMON, Gildas, "Complexification des logiques et des recompositions territoriales", in CORTÈs, Geneviève, FARET, Laurent, Les circulations transnationales..., op. cit., p. 58.

46. Simon, Gildas, " Réflexions sur la notion de champ migratoire international ", Hommes et Terres du Nord, numéro spécial, actes du colloque international, Migrations internes et externes en Europe occidentale, Lille, 16-18 octobre 1980, p. 85. 


\section{L'arrivée à Châteaubriant, Nantes et son agglomération}

\section{L'entrée en Loire-Atlantique}

Partis de provinces différentes, parcourant des trajets multiples et singuliers, ces hommes et ces femmes kurdes arrivent en Loire-Atlantique. Les dates de leur entrée en France sont indiquées dans les dossiers individuels des étrangers. Les services des préfectures s'efforcent de rendre compte de la date d'entrée en France, du point de passage utilisé par l'individu, d'indiquer s'il est entré clandestinement et s'il a déjà séjourné en France (dates et localités). Souvent, les rubriques relatives aux différents séjours passés dans d'autres départements français sont vierges; mais le contenu des dossiers permet de pallier (parfois) cette carence grâce aux cartes de séjour dont la demande implique l'obligation pour l'étranger d'attester d'un hébergement. Ces attestations datées permettent de retracer une partie des déplacements effectués par l'étranger comme ses changements d'adresse. Le tableau 1 présente les parcours d'installation de neuf Kurdes dont les dossiers ont été trouvés aux archives départementales de Loire-Atlantique. Les parcours de J., M. et Z. sont en gras.

Les récépissés de demande de cartes de séjour, les lettres adressées à l'OFPRA, les notes des renseignements généraux permettent la compréhension du contexte familial de chaque Kurde. Tous ont été hébergés dès leur arrivée en France soit par des réfugiés qualifiés de "Turcs ", soit par des membres d'associations militant contre le pouvoir en Turquie, soit par des hommes et des femmes de leur famille et/ou venant du même village. Z. est " arrivée par hasard à Angers [Maine-et-Loire] et est hébergée chez une compatriote native du même village ${ }^{47}$ ". Cette phrase équivoque interpelle par l'utilisation des termes " hasard " et " native du même village ". Pourquoi de tels propos? Z. a-t-elle voulu protéger sa compatriote? Ou alors, au regard des conditions de son voyage - cachée dans un camion -, sa venue peut résulter d'un concours de circonstances. Un mois plus tard elle arrive à Saint-Herblain. Elle y est logée par un homme, plus âgé, venant de la province de Şanliurfa; il ne porte pas le même nom de famille mais il peut s'agir d'un parent éloigné. En ce qui concerne J., il affirme que trois de ses frères résident à Nantes ${ }^{48}$ et qu'un autre frère (réfugié politique) se trouve actuellement en France ${ }^{49}$. Les a-t-il rejoints? À Nantes, il est hébergé chez un homme dont la date et le lieu de naissance sont inconnus ${ }^{50}$; il peut s'agir d'un de ses frères mais leurs noms de famille sont différents. En l'absence du dossier de cet homme, on ne peut faire que des suppositions.

47. Arch. dép. de Loire-Atlantique, 1683 W 1, fiche d'information concernant un demandeur d'asile (Z.) par la préfecture de Loire-Atlantique, Nantes, 1 p.

48. Arch. dép. de Loire-Atlantique, $1882 \mathrm{~W}$ 138, procès-verbal d'assimilation rédigé par le gardien de la paix en fonction à la BMR, 20 juin 2000, Nantes, nº 3499, 2 p.

49. Arch. dép. de Loire-Atlantique, $1882 \mathrm{~W}$ 138, lettre de J. au directeur de l'OFPRA, 16 juillet 1989, Nantes, 4 p.

50. Arch. dép. de Loire-Atlantique, $1882 \mathrm{~W}$ 138, fiche d'information concernant un demandeur d'asile (J.) par la préfecture de Loire-Atlantique, Nantes, nº 89107, 1 p. 
Coline RoNDEAU

\begin{tabular}{|c|c|c|c|c|c|c|c|c|c|}
\hline 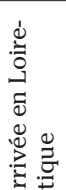 & 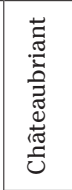 & 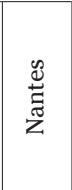 & 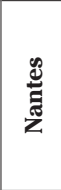 & 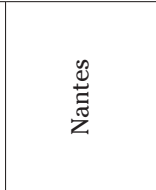 & 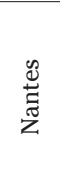 & 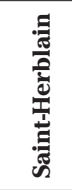 & 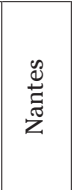 & 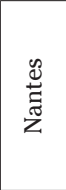 & 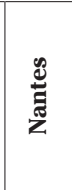 \\
\hline 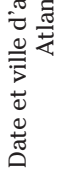 & 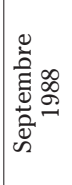 & ஓి & 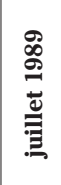 & 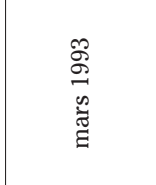 & 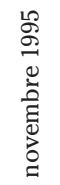 & 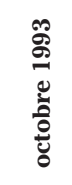 & 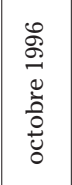 & 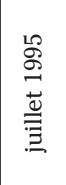 & $\begin{array}{l}\stackrel{\circ}{\circ} \\
\stackrel{\Xi}{\Xi} \\
\stackrel{\Xi}{\Xi} \\
\stackrel{\Xi}{\Xi}\end{array}$ \\
\hline 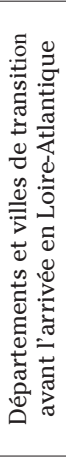 & 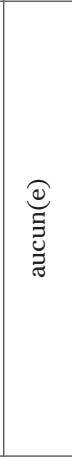 & 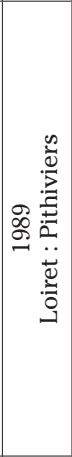 & 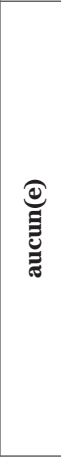 & 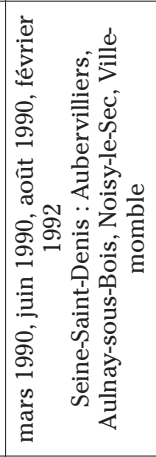 & 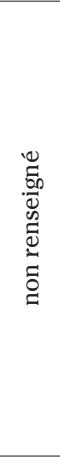 & 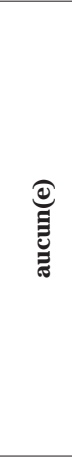 & 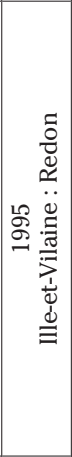 & 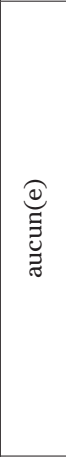 & 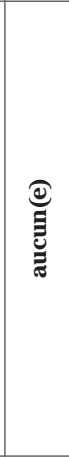 \\
\hline 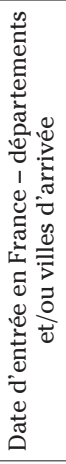 & 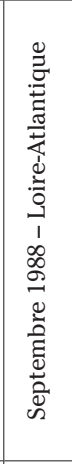 & 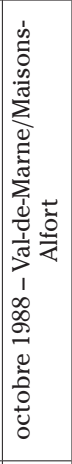 & 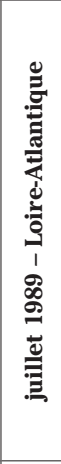 & 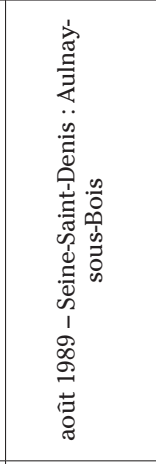 & 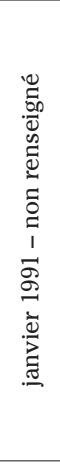 & 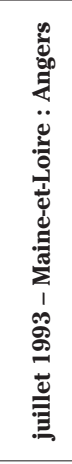 & 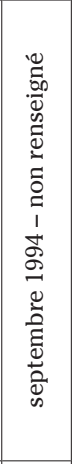 & 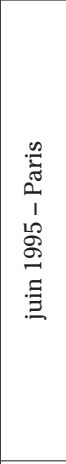 & 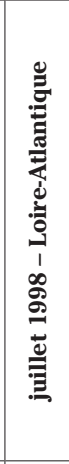 \\
\hline 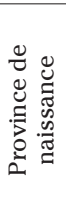 & 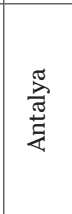 & $\frac{\stackrel{\pi}{2}}{\frac{\pi}{\pi}}$ & 氖 & 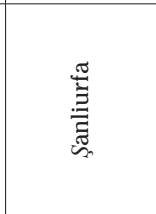 & 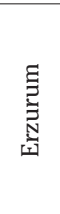 & 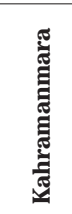 & $\begin{array}{l}\text {.్ర్ } \\
\text { స్. }\end{array}$ & $\begin{array}{l}\frac{\infty}{\mathbb{N}} \\
\frac{\pi}{I}\end{array}$ & 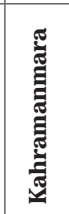 \\
\hline 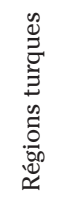 & 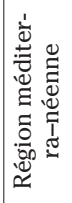 & 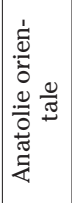 & 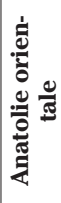 & 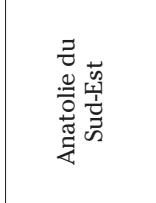 & 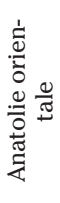 & 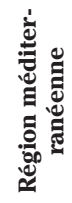 & 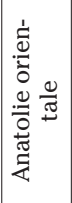 & 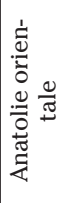 & 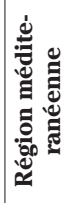 \\
\hline 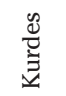 & $\dot{x}$ & $<$ & $\rightarrow$ & $\sum_{i}$ & ه & $\dot{N}$ & $\succ$ & 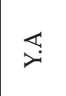 & $\dot{\Sigma}$ \\
\hline
\end{tabular}


Quant à M., il a été emmené à Nantes par ses parents en $1998^{51}$. Il ne semble pas vivre chez eux car un mois après son arrivée sur le sol français, M. est logé à Nantes chez un homme né dans la province de Malatya. Cet homme ne fait pas partie des migrants économiques puisque sur une photocopie de sa carte de résident, incluse dans son dossier, il est inscrit à la rubrique " nationalité ": "réfugié turc ${ }^{52}$ ". Il n'est pas fait mention de son origine puisque les cartes de résidence ne rendent compte que de la nationalité des requérants. Toutefois, cet homme peut être de nationalité turque et d'origine kurde. Il est probable que des réfugiés (d'origine kurde ou non) déjà installés aident leurs compatriotes. Dans sa lettre, M. explique qu'il a été aidé par des personnes déjà venues en Europe pour des activités politiques, ce qui laisse penser à l'existence de réseaux politiques et/ou ethniques en Loire-Atlantique.

Les membres des familles kurdes présents avant la décennie 1980 appartiennent aux premières générations de migrants économiques turcs. Ce sont des migrants kurdes désignés par l'administration française comme "Turcs " dans le cadre des accords de main-d'œuvre. Leur démarche témoigne d'un " savoir-faire " face à l'administration : celui d'effacer leur origine, de la rendre invisible afin de mettre en avant leur nationalité et de pouvoir ainsi quitter la Turquie. Dès lors, la possibilité de les retrouver dans les archives au cours de la décennie 1970 est nulle. "L'identification de la nationalité des personnes devient un aspect décisif du travail juridicobureaucratique de catégorisation. Comme on ne peut déterminer la nationalité d'une personne que par référence à son lieu de naissance et à ses [ascendants], c'est en fait toute l'identité civile des individus qui devient l'objet central des entreprises étatiques d'identification ${ }^{53}$ ". Cette invisibilité a été choisie par les Kurdes afin de pouvoir quitter le territoire turc. Mais elle est aussi subie, car affirmer leur nationalité était la seule manière légale de migrer et de s'installer en France. Ainsi, l'arrivée de demandeurs d'asile peut permettre de retrouver des Kurdes arrivés dans la décennie 1970 en retraçant, à partir des sources, leur arbre généalogique.

Compte tenu du laps de temps entre leur entrée en France et la rédaction de leur lettre de demande d'asile adressée à l'OFPRA, ces Kurdes ont nécessairement été aidés par leurs hébergeurs. Ces derniers apportent une aide matérielle confirmée dans les attestations d'hébergement mais aussi un soutien moral malheureusement indétectable. Z. rédige une lettre, de deux pages, onze jours après son arrivée à Angers; sa lettre est la seule à être dactylographiée. Z. est issue "d'une famille kurde très pauvre ", - ses parents sont " agriculteurs " et elle est " éleveuse "-, il est donc peu

51. Arch. dép. de Loire-Atlantique, 1882 W 142, lettre de M. au directeur de l'OFPRA, 7 août 1998, Nantes, p. 2.

52. Arch. dép. de Loire-Atlantique, 1882 W 142, photocopie de carte de résidence produite par la préfecture de Loire-Atlantique, novembre 1989, Nantes, 1 p.

53. NoIRIEL, Gérard, "Représentation nationale et catégories sociales. L'exemple des réfugiés politiques ", Genèses, 1977, vol. 26, nº 1, p. 44. 
probable que la machine à écrire lui appartienne ${ }^{54}$. Sa lettre ne comporte aucune faute d'orthographe ni de conjugaison et elle utilise des mots de liaison. La compatriote qui l'héberge a dû l'aider à rédiger cette lettre et lui prêter une machine à écrire. Ou alors, son hôte a été un relais avec une association d'aide aux étrangers à Angers.

Dans le dossier de J., une correspondance entre son avocat et la préfecture de Loire-Atlantique ainsi qu'une lettre de recours rédigée par l'avocat et adressée à la CRR ont été conservées ${ }^{55}$. D'après les sources, il est le seul à recourir à un avocat ${ }^{56}$. La présence de telles sources est rare puisque sans la correspondance entre la préfecture de Loire-Atlantique et l'avocat, la lettre de recours à la CRR n'aurait pas été conservée car il s'agit d'une photocopie adressée en pièce jointe au cours de cette correspondance. Les échanges entre l'avocat de J. et la préfecture dévoilent les complications auxquelles font face les demandeurs d'asile. Vulnérables dans leur parcours d'installation et administratif, ne maîtrisant pas la langue française, ne connaissant pas les rouages de l'administration, ces hommes et ces femmes ont dû être dépassés par des démarches qui impliquent l'accumulation d'attestations de recours, d'hébergement, de revenus, de scolarité, de mariage... En effet, " au cours de sa phase de transition, l'étranger ne considère pas du tout ce modèle [culturel] comme un asile protecteur, mais bien plutôt comme un labyrinthe dans lequel il a perdu tout sens de l'orientation ${ }^{57}$ ".

\section{Le temps de l'installation}

Qu'ils obtiennent ou non la qualité de réfugié, les Kurdes continuent de circuler. Au fil des déplacements, un certain ancrage identitaire apparaît, à la fois pour les Turcs et les Kurdes, par la création notamment d'associations promouvant respectivement ces deux peuples en Loire-Atlantique : association islamique turque, association culturelle de l'Islam des travailleurs turcs, parti communiste de Turquie marxiste-léniniste ${ }^{58} \ldots$ En reliant

54. Arch. dép. de Loire-Atlantique, 1683 W 1, formulaire de demande de statut de réfugié de Z., [non daté], 12 p.

55. Arch. dép. de Loire-Atlantique, 1882 W 138, lettre de l'avocat de J. au président de la CRR, 25 juillet 1990, Paris, 4 p.; 1882 W 138, lettre de l'avocat de J. au préfet de LoireAtlantique, 14 août 1990, Paris, 1 p.; 1882 W 138, lettre du secrétaire général de la direction de la réglementation du $2^{\mathrm{e}}$ bureau du cabinet du préfet de Loire-Atlantique à l'avocat de J., 13 septembre 1990, Nantes, 1 p.; 1882 W 138, lettre de l'avocat de J. au secrétaire général de la direction de la réglementation du $2^{\mathrm{e}}$ bureau du cabinet du préfet de Loire-Atlantique, 28 septembre 1990 , Paris, 1 p.

56. Cela ne veut pas dire que les autres Kurdes n'en ont pas eu la possibilité, sachant que des avocats plaident à la CRR.

57. SсHÜTZ, Alfred, L'Étranger. Un essai de psychologie sociale, Paris, Allia, 2010, p. 38.

58. Arch. dép. de Loire-Atlantique, 1505 W 155, note du ministère de l'Intérieur et de la décentralisation (direction générale de la police et direction centrale des renseignements généraux - DGP et DCRG) au préfet de Loire-Atlantique et au directeur des renseignements généraux, 18 janvier 1984, Nantes, n 24-292, "Colonie turque de Nantes ", 3 p.; 1260 W 191, note du ministère de l'Intérieur et de la décentralisation (DGP et DCRG) 
les données des dossiers individuels des Turcs et des Kurdes, il est possible d'identifier des points de rencontre et des connexions à une échelle locale. Les changements d'adresse sont une forme visible des circulations.

Les données recueillies mettent en évidence différents quartiers privilégiés par les Turcs et les Kurdes à Nantes. En effet, les quartiers administratifs, Bellevue - Chantenay - Sainte Anne et Dervallières - Zola, concentrent la majorité des adresses temporaires ou fixes. Le premier oppose une partie pavillonnaire à des grands ensembles et des zones industrielles sur les rives de la Loire. Bellevue - Chantenay - Sainte Anne concentre une " forte proportion de personnes non diplômées " et des personnes " détenant des diplômes de niveau CEP, CAP, BEPC ou BEP ". Les CSP (catégories socio-professionnelles) les plus représentées en 1999 dans ce quartier sont les chauffeurs, les ouvriers non qualifiés de type artisanal, les ouvriers qualifiés de type artisanal, les ouvriers non qualifiés de type industriel. Quant à Dervallières - Zola, il est caractérisé par sa diversité sociale grâce à la répartition entre " micro-quartiers d'habitat HLM " et zones résidentielles ${ }^{59}$. Ces deux quartiers sont proches de la commune de Saint-Herblain où la zone industrielle mobilise de nombreux ouvriers turcs et kurdes aux Chantiers Modernes, à la fonderie Ribrac Gendron \& Josse. Le centre-ville et Malakoff - Saint-Donatien sont deux quartiers où les Kurdes (dont J.) se sont installés afin d'être à proximité de leurs restaurants ${ }^{60}$. L'installation d'un commerce dans le centre-ville de Nantes est rendue logique par la présence du centre historique de Nantes, d'un pôle universitaire et hospitalier engendrant beaucoup de circulations estudiantines et touristiques. Enfin, le quartier Nantes-Erdre concentre une part importante de Turcs et de Kurdes. Ce quartier est à proximité de la zone industrielle de Carquefou où les ouvriers travaillent dans des sociétés de galvanisation, de menuiserie, etc. Les CSP les plus représentées en 1999 sont les ouvriers non qualifiés de type industriel, les ouvriers qualifiés de type industriel, les techniciens ${ }^{61}$.

Les Kurdes se situent en majorité aux abords des communes de SaintHerblain et de Carquefou. Leurs différents déplacements montrent que leurs premières adresses sont des points de chute qui se caractérisent par

au préfet de Loire-Atlantique et au directeur des renseignements généraux, 4 octobre 1984, Nantes, n 454-5264, "Turcs ", 2 p.; 1944 W 133, note des renseignements généraux, octobre $1991, \mathrm{n}^{\circ} 76,1 \mathrm{p}$.

59. [http://www.insee.fr/fr/insee_regions/pays-de-la-loire/themes/dossiers/dossier06. pdf] consulté le 2 juin 2016.

60. Arch. dép. de Loire-Atlantique, 1882 W 138, rapport d'un sous-brigadier de police en fonction à la BMR du contrôle de l'immigration en Loire-Atlantique au commandant de police et chef de la BMR de Loire-Atlantique, 23 juin 2000, Nantes, $n^{\circ} 349$, "Demande d'enquête : naturalisation par mariage art. 21.20 du code civil ", 3 p.; 1882 W 38, rapport d'un sous-brigadier de police en fonction à la BMR du contrôle de l'immigration en LoireAtlantique au commandant de police et chef de la BMR de Loire-Atlantique, 28 août 2000, Nantes, no 00/092, "Demande d'enquête : naturalisation par mariage art. 21.20 du code civil ", 3 p.

61. [http://www.insee.fr/fr/insee_regions/pays-de-la-loire/themes/dossiers/dossier06. pdf] consulté le 2 juin 2016. 
la courte durée d'installation en ce lieu et la présence d'un(e) hébergeur(se). Tous ont rejoint à un moment donné la ville de Nantes en intégrant des foyers de la SONACOTRA (Société nationale de construction de logements pour les travailleurs) ou par l'ouverture d'un commerce (restauration rapide). Certaines adresses de demandeurs d'asile kurdes sont à proximité de lieux habités par des migrants économiques turcs qui pourraient être d'origine kurde. Tel est le cas à Châteaubriant où le recoupement des sources a permis de rendre visible l'origine kurde de certains travailleurs turcs $^{62}$.

Après leur entrée en France, la grande majorité des hommes turcs est arrivée directement à Châteaubriant pour signer des contrats de travail. Les sources démontrent qu'ils n'ont pas quitté la ville. Leurs changements d'adresse relèvent pour la plupart des cas d'une installation dans un logement plus grand afin de faire venir leurs familles. Trois rues sont majoritairement habitées par les ressortissants turcs dans les années 1970-1980 : rue des Tanneurs, rue Armand Franco et faubourg de Béré. Ceci s'explique par la présence de la fonderie Huard qui a recruté et logé de nombreux travailleurs étrangers, portugais puis turcs. La majorité des Turcs travaillait en tant qu'ouvriers fondeurs, ouvriers spécialisés en fonderie, ébarbeurs, chauffeurs de four, maçons au sein des fonderies Huard. Mais ils étaient aussi présents dans la construction mécanique au sein de l'entreprise Huard-UCF-SCM et dans les travaux agricoles de l'entreprise MEAC SA. Le recoupement des sources, la reconstitution d'arbres généalogiques attestent la présence de Kurdes travaillant aux fonderies Huard. Mais le nombre de réfugiés kurdes installés à Châteaubriant n'est guère important, ce qui montre que la présence potentielle de migrants économiques kurdes y est minime, voire dissimulée. L'étude des circulations des migrants économiques kurdes à Nantes et à Châteaubriant (voire ailleurs) au cours de la décennie 1970 est quasiment chimérique. Pourtant, le dossier d'un couple de Kurdes habitant à Rennes a été trouvé aux archives départementales d'Ille-et-Vilaine. Ce couple a fait une demande de naturalisation auprès de la préfecture d'Ille-et-Vilaine ${ }^{63}$. Auparavant, ils étaient installés à Châteaubriant et hébergés par la famille de la femme. Cette famille avait été catégorisée, par la préfecture de Loire-Atlantique, en tant que migrants économiques turcs et non en tant que Kurdes ${ }^{64}$. Les demandes de naturalisation dans un autre département permettent, en plus de l'arrivée de demandeurs d'asile, de retrouver ces « Kurdes invisibles".

62. Lettre d'un réfugié kurde (X.) affirmant la présence de sa sœur à Châteaubriant : Arch. dép. de Loire-Atlantique, 1882 W 127, lettre de X. au directeur de l'OFPRA, 26 septembre 1988, Châteaubriant, 1 p.; Le dossier de la sœur de X. a été retrouvé. Cette femme, arrivée dans la décennie 1970, était considérée comme turque : 1286 W 271, Dossiers individuels d'étrangers (1959-1988).

63. Arch. dép. d'Ille-et-Vilaine, 2752 W 11, Naturalisation par décret de O. par le ministère de l'Intérieur, 18 avril 2003, Rennes, 2 p.

64. Arch. dép. de Loire-Atlantique, 1286 W 162, Dossiers individuels d'étrangers (19601988). 


\section{Retourner au pays?}

«Le réfugié ne veut pas quitter son pays, il y est contraint parce que sa vie est en danger; il n'est donc pas préparé au départ qu'il vit comme une injustice et l'interdiction de retourner dans son pays en fait une victime de ce bannissement ${ }^{65}$ ". Car d'après la section C. art. 1 de la Convention de Genève, la protection cesse "si [la personne réfugiée] est retournée volontairement s'établir dans le pays qu'elle a quitté ou hors duquel elle est demeurée de crainte d'être persécutée ${ }^{66} "$. Lors de la consultation des procès-verbaux d'assimilation, une question est posée aux Kurdes : « Se rend-il encore régulièrement [dans son pays d'origine]? " Toutes les réponses sont négatives. Aucun d'entre eux ne serait reparti, excepté un homme qui est retourné en Turquie pour se marier. Mais puisque les sources s'arrêtent à un moment de leur vie et qu'aucune trace d'eux n'a été trouvée par la suite, il est possible qu'ils y soient retournés ${ }^{67}$. M. est le seul à avoir fait le choix, après avoir effectué toutes les démarches pour obtenir l'asile politique, de retourner définitivement en Turquie. Sept mois après l'enregistrement de sa demande auprès de l'OFPRA, un courrier informe que M. s'est désisté. Six jours plus tard, il quitte le territoire français en prenant un avion à l'aéroport Nantes-Atlantique à destination d'Istanbul ${ }^{68}$. D'après sa lettre adressée à l'OFPRA, ses parents vivent à Nantes et il est célibataire. Pourquoi est-il retourné en Turquie? Son retour est-il lié à l'arrestation d'Öcalan, le 15 février 1999, qui met fin à la lutte armée? A-t-il décidé d'aider politiquement son pays? Est-il parti pour aider des membres de sa famille? Sans sa lettre de désistement, seules des hypothèses peuvent être énoncées.

Lors du 30 avril 2016, journée dédiée aux femmes organisée par Zin 44, nous avons rencontré une réfugiée politique kurde (B.). Installée depuis neuf ans en France avec son mari, elle n'est pas retournée en Turquie. Car B. a peur d'être reconnue et attrapée par la police turque puisqu'elle a combattu auprès de la guérilla kurde. Son souhait est de vivre dans son pays et de retrouver sa famille dont ses parents : " La France c'est bien, c'est un beau pays. Mais la Turquie... c'est mon pays. " L'absence de ses proches

65. Chevallier, Corinne, "Itinéraire d'une communauté en exil : les réfugiés politiques turcs de gauche en France après le coup d'état militaire de septembre 1980 en Turquie ", op. cit., p. 53.

66. [http://www.ohchr.org/FR/ProfessionalInterest/Pages/StatusOfRefugees.aspx] consulté le 15 février 2017.

67. Arch. dép. de Loire-Atlantique, 1882 W 30, procès-verbal d'assimilation rédigé par le sous-brigadier de la police en fonction à la BMR de Loire-Atlantique, 16 mars 2000, Nantes, p. 2.; $1882 \mathrm{~W} 138$, procès-verbal d'assimilation rédigé par le gardien de la paix en fonction à la BMR, 20 juin 2000, Nantes, nº 3499, p. 2.; 1882 W 38, procès-verbal d'assimilation rédigé par le sous-brigadier de police en fonction à la BMR de Loire-Atlantique, 10 août 2000, Nantes, n 393 , p. 2.; 1882 W 138, procès-verbal d'un agent administratif de la mairie de Nantes au directeur départemental des Affaires Sanitaires et Sociales, ${ }^{\mathrm{er}}$ septembre 1993, Nantes, p. 2.

68. Arch. dép. de Loire-Atlantique, 1882 W 142, Lettre du directeur départemental de la police aux frontières de Loire-Atlantique à la préfecture de Loire-Atlantique, 12 avril 1999, Nantes, no 359, " Départ ressortissant turc ", 1 p. 
lui pèse et pour pallier cette barrière terrestre, elle communique avec eux par les réseaux sociaux (Skype, Facebook) et s'informe de l'actualité turcokurde. D'une certaine manière, le contact maintenu avec sa famille et ses amis permet à B. d'" aller et venir " entre la Turquie et la France. Les réseaux sociaux forment une toile d'araignée gigantesque permettant aux réfugiés et à leurs familles de construire un nouvel espace de sociabilité.

Les circulations mettent en exergue des mobilités, des choix de quartiers à travers le temps. Néanmoins, l'étude de ces circulations ne permet pas d'aborder tout le processus décisionnel : pourquoi tel individu déménage-t-il? Pourquoi s'installe-t-il à tel endroit? Pourquoi part-il? De plus, saisir la nature des relations entre ces Kurdes et les premières générations de migrants est complexe. Seules des hypothèses peuvent être énoncées sur de possibles liens familiaux, des rapprochements entre voisins (proximité spatiale) ou entre collègues de travail (fonderies Huard). Ces histoires de Kurdes s'entremêlent avec celles de migrants économiques turcs à cause de leurs parcours migratoires, leurs circulations au sein du département. Mais quand et comment apprend-on que tel individu est un Kurde? Qui l'écrit et comment? Comment expriment-ils leur identité? Dans le cadre du CCK de Nantes, de quelle(s) manière(s) ces hommes et femmes prennent-ils part au développement voire à l'affirmation de leur identité?

\section{Kurdicité et territoire}

" On trouve parmi les Kurdes un noyau dont l'identité est sans ambiguïté kurde et, tout autour, une masse fluide de degrés divers de kurdicité, des individus qui sont quelque chose d'autre en même temps que Kurdes, et qui, selon les circonstances, peuvent accentuer ou minimiser leur identité kurde $^{69}$ ». En s'interrogeant sur l'identité kurde, nous questionnons la représentation des Kurdes par l'administration mais aussi les moyens dont ils disposent pour s'affirmer. Quels sont leurs référents identitaires? Comment s'approprient-ils ce nouveau territoire?

\section{Être Kurde au regard des sources administratives}

Au cours des années 1980, des notes du ministère de l'Intérieur adressées à la préfecture de Loire-Atlantique rendent compte de mouvements militants à Nantes. C'est ainsi que la plupart des militants d'extrême gauche sont catégorisés comme étant d'origine kurde et de confession chiite ${ }^{70}$. L'affirmation de Kurdes de confession chiite suggère la présence de Kurdes d'Iran, d'Irak du sud et/ou d'Alévis de Turquie à Nantes. Ces militants appartiendraient au TDKP (Türkiye Devrimci Komünist Partisi) fondé en 1978 en

69. VAN BRUINESSEN, Martin, " Nationalisme kurde et ethnicités intra-kurdes ", Les Kurdes et les États, Peuples Méditerranéens, juillet-décembre 1994, nº 68-69, p. 13.

70. Arch. dép. de Loire-Atlantique, 1260 W 191, note du ministère de l'Intérieur et de la décentralisation (DGP-DCRG) au préfet de Loire-Atlantique et au directeur des renseignements généraux, 4 octobre 1984, Nantes, n 454-5264, " Turcs ", 2 p. 
Turquie. Ces militants ne sont pas les seuls à Nantes. Ils s'opposent régulièrement aux militants d'extrême droite sunnites. Les rivalités politiques s'imbriquent avec les tensions religieuses pour l'administration française. Mais les Kurdes sont des deux confessions, et en majorité sunnites. Les renseignements relayés par les autorités françaises rendent compte de l'existence de Kurdes seulement lors de frictions avec les Turcs. Il s'agit de dresser le tableau d'un ensemble d'individus (" la colonie turque ") sans s'attarder sur des disparités plus profondes.

Un parallèle peut être effectué avec les certificats de l'OFPRA qui informent les individus de la reconnaissance de leur qualité de réfugié. L'Office ne qualifie pas les réfugiés kurdes de la même manière. Certains sont des " réfugiés de nationalité turque d'origine kurde ", "réfugiés de nationalité turque " ou " réfugiés d'origine kurde ${ }^{71}$ ". Pourquoi une telle différenciation? La première dénomination associe la nationalité (lieu de naissance) et l'ethnie (origine). La seconde ne mentionne que la nationalité en niant l'origine. Quant à la dernière, l'absence de la nationalité laisse croire que le réfugié est apatride. Quelle logique est sous-jacente à ces dénominations? Comment les analyser? Que signifient-elles? Pourquoi un Kurde menacé en Turquie à cause de son origine obtient-il la qualité de réfugié mais sous le titre de "réfugié de nationalité turque "? Ces disparités interrogent sur la reconnaissance du fait d'être Kurde en France et d'être réfugié kurde. Qu'en est-il pour les Kurdes? Minimisent-ils ou accentuent-ils leur kurdicité ? Quand et comment l'affirment-ils?

\section{Quand les Kurdes s'identifient}

Les lettres des demandeurs d'asile répondent à une attente de l'administration française. Ils doivent prouver, par tous les moyens, que leur vécu est relatif à l'art. 1 de la convention de Genève et au protocole de New York de 1967. Gérard Noiriel explique que ces demandeurs d'asile " accentuent la dimension "humaine" de leur expérience en cherchant par tous les moyens à apitoyer les juges " et ajoute que " l'exemple des réfugiés illustre parfaitement la double logique d'assujettissement que Michel Foucault plaçait au cœur de ses analyses sur "l'étatisation des relations de pouvoir" 72 ". Dès lors, comment étudier ces lettres de demandeurs d'asile? Comment examiner leur rapport à leur identité kurde, compte tenu des tenants et aboutissants de telles lettres?

Les hommes kurdes n'écrivent pas qu'ils sont "Kurdes" dans leurs lettres de demande d'asile. J. n'écrit pas dans sa lettre qu'il est Kurde. Il mentionne plusieurs termes : "Kurdistan ", " le but c'est l'assimilation des

71. Arch. dép. de Loire-Atlantique, 1882 W 127, certificat de l'OFPRA à X., 3 septembre 1990, Fontenay-sous-Bois; 1882 W 138, certificat de l'OFPRA à J., 4 avril 1991, Fontenaysous-Bois; 1882 W 38, certificat de l'OFPRA à Y., 29 mars 1996, Fontenay-sous-Bois.

72. NoIRIEL, Gérard, État, nation et immigration. Vers une histoire du pouvoir, Paris, Belin, 2001, p. 287. 
Kurdes ", "ils nous imposaient la politique turque ", " nous étions le seul village kurde ${ }^{73}$ ». Le " nous " est utilisé pour dénoncer les politiques coercitives du gouvernement turc à l'encontre des Kurdes. J. déclame sa kurdicité en se référant à l'espace dans lequel il se situe : son village est au Kurdistan et non en Turquie (origine spatiale). L'identité kurde de J. passe par sa reconnaissance de l'existence de ce territoire ainsi que de ses activités militantes précédemment évoquées. Sa kurdicité transparaît également par la pratique des langues kurde et turque avec sa famille et ce, onze ans après son arrivée en France ${ }^{74}$. Quant à M., il dit qu'il est d'origine kurde. Cette déclaration intervient pour expliquer pourquoi il a été licencié. La revendication de son origine semble ne pas être un marqueur essentiel pour se décrire en tant qu'individu. Néanmoins, divers passages montrent que sa kurdicité s'est développée au fil des années. Des moments de sa vie ont provoqué sa participation à des activités politiques pour défendre la cause kurde : "j'ai passé mon enfance au milieu du peuple kurde maltraité et humilié ", " en compagnie des personnes qui venaient du Kurdistan ", "les persécutions que subissaient les Kurdes au Kurdistan ${ }^{75}$ ". Il s'est construit à travers ses rencontres, ses actions. Il est né au sein d'une famille kurde et a appris à être kurde à sa manière. Enfin, Z. déclare : " Je suis Kurde ${ }^{76}$. " Elle n'utilise pas les mots " d'origine " ni de "nationalité turque ». Z. se différencie des hommes par sa manière d'exposer sa kurdicité. Elle affirme être kurde et explique ensuite ce qu'elle a subi à cause de son origine ethnique. Elle ne sous-entend rien, elle ne laisse pas de place au doute. Son comportement se rapproche de celui des femmes rencontrées au CCK de Nantes.

Le 30 avril 2015, une journée dédiée exclusivement aux femmes au CCK de Nantes a été organisée. Une Kurde s'est présentée à l'assemblée par les termes suivants : "Je suis Kurde, je suis une femme. " L'identité kurde est affirmée au préalable puis lui est associée l'identité féminine. Ces femmes sont en effet soumises à une double discrimination : être Kurde et être femme. Leur combat unit la reconnaissance de la cause kurde et celle de la libération des femmes kurdes. Leur engagement est le fruit du développement de théories politiques d'Öcalan au sein du PKK. Ces femmes défendent la réappropriation de leur corps, leur autonomie, leur sexualité et dénoncent les violences physiques, les oppressions, etc. Une dialectique identité et genre s'engage alors pour ces dernières. Ces femmes enseignent à leurs enfants les valeurs et les coutumes kurdes, auxquelles est associé l'apprentissage des langues kurde et française au sein du centre. L'enseignement du kurde permet de resserrer les liens de cette « commu-

73. Arch. dép. de Loire-Atlantique, 1882 W 138, lettre de J. au directeur de l'OFPRA, 16 juillet 1989, Nantes, 4 p.

74. Arch. dép. de Loire-Atlantique, 1882 W 138, procès-verbal d'assimilation rédigé par le gardien de la paix en fonction à la BMR, 20 juin 2000, Nantes, nº 3499, p. 1.

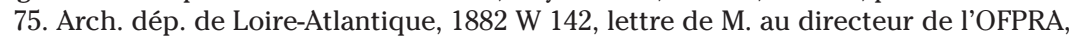
7 août 1998, Nantes, 2 p.

76. Arch. dép. de Loire-Atlantique, $1683 \mathrm{~W}$ 1, formulaire de demande de statut de réfugié de Z., p. 12. 
nauté kurde » de Nantes puisque certains ne pratiquent que le turc. En effet, lors de cette journée, la porte-parole de Zin 44 servait de traductrice; elle passait du français au turc, ce qui sous-entend que certaines femmes ne parlaient pas le kurde. Cette traduction, sur le long terme, montre la volonté de ne pas exclure de l'échange celles qui ne connaissent ni le français ni le kurde. Ces femmes portaient des vêtements de tradition kurde (les plus âgées) ou "à l'européenne " (les plus jeunes). Néanmoins, comme l'explique Chirine Mohseni, "les Kurdes se distinguent toujours des Français par leur manière de porter les vêtements et par le choix des formes et des couleurs ". En effet, les vêtements étaient colorés, larges, affirmant " une stratégie menée par l'étranger pour se montrer intégré aux yeux de l'autre, tout en persévérant discrètement son identité en s'attachant à des normes qui sont a priori invisibles pour l'autre ${ }^{77}$ ".

\section{L'appropriation d'un territoire : pratiques et moyens}

Évoquer la représentation et le développement de l'identité kurde implique de s'interroger sur l'adaptation de celle-ci au sein du territoire nouveau qu'est la Loire-Atlantique. Plusieurs Kurdes ont participé à des stages d'alphabétisation, dont J. qui a été aidé par le mouvement d'éducation populaire «Culture et Liberté " à Rezé. Par la suite, ces hommes se sont mariés avec des Françaises, ce qui leur a permis de régulariser leur situation. Leurs parcours invitent à se demander si ces hommes brident pour un temps leur identité kurde afin d'acquérir la nationalité française ou si au contraire ils étouffent complètement leur identité kurde? En l'absence de rencontres avec eux, il est impossible de le savoir car les sources cessent au moment de la naturalisation. Cette piste de réflexion montre l'intérêt de contacter ces hommes afin d'apporter un complément à l'histoire de leurs parcours et de découvrir ce qu'ils sont devenus. Mais il serait trompeur de penser obtenir un récit identique, par rapport aux sources étudiées, car ces Kurdes raconteraient leur passé au regard de qui ils sont aujourd'hui. Une forme de reconstruction du vécu serait mise en œuvre. Ainsi, Z., âgée de 44 ans aujourd'hui, n'est plus celle qui est arrivée en France à l'âge de 19 ans. Dès lors, leurs positionnements, par exemple à l'égard de leur identité kurde, ont fluctué et évolué. Les rencontres avec les Kurdes du CCK de Nantes permettent (d'une certaine manière) de se rendre compte aujourd'hui de leur adaptation à la société française et de percevoir la façon dont ils lient leur identité à leur nouveau mode de vie. Par quels moyens s'approprient-ils ce nouveau territoire?

Le centre culturel kurde propose à la fois des cours de kurde et de français. Cet apprentissage facilite l'installation et l'adaptation sur le territoire français. En effet, ces hommes et ces femmes peuvent utiliser les transports en commun ainsi que d'autres moyens de locomotion qui nécessitent de

77. MohsEnI, Chirine, "Les réfugiés kurdes en France : intégration et modes de vie ", Revue de France Terre d'Asile, septembre 1999, nº 2, p. 15-16. 
savoir lire le français. La capacité de se déplacer favorise la recherche d'un emploi et ainsi l'octroi d'un revenu permettant d'obtenir un logement. En parallèle à cet enchaînement empirique, savoir parler français permet aux Kurdes de faire entendre leurs revendications dans la sphère publique : dénonciation de la politique turque à l'encontre des Kurdes, demande de la libération d'Öcalan, de la paix au Kurdistan, de la libération des députés du HDP (Halkların Demokratik Partisi), etc. Ils participent chaque année à la manifestation du $1^{\mathrm{er}}$ mai, ce qui questionne leur rapport à la citoyenneté et leur engagement associatif. Leurs manifestations sont relayées par la presse locale et leurs porte-parole peuvent exprimer les raisons de leurs démarches ${ }^{78}$. Ils utilisent également d'autres moyens tels que les réseaux sociaux et des sites internet (magazine Kedistan) afin de transmettre leurs actions et d'inviter un maximum de personnes. Chaque année, les Kurdes de Nantes défilent le(s) 20 et/ou 21 mars (premier jour du printemps) lors du Newroz ${ }^{79}$. Ils utilisent l'espace public afin de mettre en avant leur histoire et se retrouver ensemble pour célébrer cet évènement. Ils y brandissent des luminaires, des drapeaux du Kurdistan et des différentes forces armées kurdes tels que celui de l'YPG (Yekîneyên Parastina Gel). Ces drapeaux sont des référents identitaires pour ces Kurdes. Clémence ScalbertYücel explique que " si les Kurdes ont leur drapeau, c'est qu'ils forment une nation comme les autres : chaque nation et chaque État a son drapeau. Le drapeau est le symbole de l'identité et de son existence ${ }^{80}$ ". Dès lors, les Kurdes sont, au regard de la théorie d'Anthony Smith, une « ethnic community " qui a conscience " d'être un groupe historique " doté d'un sentiment d'unité ${ }^{81}$. Celui-ci s'affiche dans l'espace public mais aussi dans l'enceinte

78. Plusieurs dépêches sur le site de Ouest-France (Pays de la Loire, Nantes; Bretagne, Rennes) tels que : [http://www.ouest-france.fr/pays-de-la-loire/nantes-44000/manifestation-de-kurdes-dans-les-rues-de-nantes-ce-mercredi-10-fevrier-4028282] ; [http://www. ouest-france.fr/pays-de-la-loire/nantes-44000/nantes-nouvelle-manifestation-prokurdedans-le-centre-ville-4606984]. Mais aussi sur Presse Océan (Nantes, Saint-Nazaire, LoireAtlantique) comme : [http://www.presseocean.fr/actualite/nantes-une-centaine-de-manifestants-kurdes-dans-les-ru es-04-11-2016-208584].

79. Pour les Kurdes l'origine du Newroz se réfère à la révolte du forgeron Kawa contre le tyran Dehak. Özcan Yilmaz explique que "le mythe de Kawa est emprunté à la mythologie iranienne, notamment au Shahnameh (Livre des Rois) de Ferdowsi. Selon la mythologie iranienne, Newroz (21 mars) correspond au jour de l'intronisation du roi Jamshid. Cependant, l'orgueil de celui-ci attire les foudres du dieu qui, pour le punir, envoie Dehak. Ce jeune Arabe, en collaboration avec le diable, sème le chaos dans le pays de Jamshid et prend son trône. En plus des injustices qu'il fait subir, il doit sacrifier des enfants aux serpents qui apparaissent sur ses épaules. Cela provoque un soulèvement. Un forgeron du nom de Kawa le bat et l'enferme dans le mont Damavand. À partir du Xvi ${ }^{\mathrm{e}}$ siècle, un autre mythe fait remonter l'origine des Kurdes à un peuple formé des descendants des enfants qui, pour échapper à Dehak, se sont réfugiés dans les montagnes. Dans les années 1930, un nouveau récit insiste sur l'origine kurde du mythe de Kawa [...]. Le Newroz signifierait ainsi le soulèvement des Kurdes, dirigés par Kawa, contre le roi assyrien Dehak et la destruction de l'Empire assyrien en 612 av. J-C par les Mèdes, considérés comme des ancêtres des Kurdes ", in YILMAZ, Özcan, La formation de la nation kurde... op. cit., p. 51.

80. SCALBERT-YÜCEL, Clémence, "Kurdes sans Kurdistan ", Outre-Terre, n 12, 2005, p. 104.

81. Cité par YILMAZ, Özcan, La formation de la nation kurde..., op. cit., p. 7. 
du CCK par la présence des portraits d'Abdullah Öcalan (1949-...), Sakîne Cansiz (1958-2013), Mazlum Doğan (1955-1982), Fidan Doğan (1982-2013), Leyla Söleymez (1989-2013), Kemal Pir (1952-1982), etc. Ce sont de véritables symboles liés aux combats qu'ils ont menés. Leader du peuple kurde, Apo est, depuis 1999, emprisonné seul sur l'île d'Imrali ; il a été condamné à mort en 1999 pour avoir trahi la nation turque, mais sa peine fut commuée en prison à vie en 2002. Quant aux autres militants de la cause kurde, ils sont considérés comme des "martyrs", des hommes et des femmes "valeureux " selon le porte-parole du CCK. Ce dernier ajoute même qu'ils sont fiers d'avoir accroché leurs portraits dans leur centre ${ }^{82}$. Ces militants ont mené des grèves de la faim (entraînant leur mort) dans la prison de Diyarbakir en 1982, d'autres se sont immolés ou se sont pendus. Quant à Sakîne Cansiz, Fidan Doğan, Leyla Söleymez, elles ont été assassinées à Paris dans la nuit du 9 au 10 janvier 2013.

Deux niveaux de temporalité sont à souligner, à savoir l'" espace-temps des déménagements caractéristiques d'un cycle de vie individuel et familial, conquête groupale des territoires de référence " et l'" espace-temps des migrations ${ }^{83}$ ". Ces migrations ont dévoilé une rupture temporelle entre les migrations économiques et celles des demandeurs d'asile, en lien avec le contexte en Turquie et la politique migratoire en France. Cet " espacetemps des migrations "s'imbrique dans deux territoires (la Turquie et la France). La dimension de transnationalité apporte à ces migrations une vision dynamique, pour saisir les stratégies migratoires mises en œuvre par ces Kurdes (transports utilisés, pays traversés, réseaux familiaux/ politiques, points de passage...). Par la suite, le passage du transnational au local (" espace-temps des déménagements") a permis de retracer une partie des parcours, les installations nouvelles ou répétées des Kurdes en Loire-Atlantique (quartiers privilégiés, entreprises employant des Turcs et Kurdes, réseautage...).

Une autre manière d'aborder l'" espace-temps " a été développée par les rencontres avec les Kurdes du CCK. Leurs participations à des manifestations/fêtes annuelles, leurs rencontres hebdomadaires au sein du centre mettent en exergue les liens entre utilisation du territoire et réactivation des liens identitaires. La manière dont ils expriment leur identité kurde a ainsi été développée par leur mobilité. Cette identité s'est aussi caractérisée par la possession de drapeaux, l'utilisation et l'apprentissage de la langue kurde. Ils exposent leur kurdicité, à la fois à l'intérieur de leur "communauté " et à l'extérieur. En 2016, l'un des porte-parole du CCK faisait part

82. Rencontre avec l'un des porte-parole du CCK à Nantes, 22 février 2017, 50 minutes. Pour information, le CCK a été incendié dans la nuit du 8 avril 2017, voir l'article de OuestFrance sur cet événement : [http://www.ouest-france.fr/pays-de-la-loire/nantes-44000/ video-les-images-du-centre-culturel-kurde-incendie-saint-herblain-4918089].

83. TARrius, Alain, Les nouveaux cosmopolitismes. Mobilités, identités, territoires, Paris, 2000, p. 39-42. 
de l'envie de créer plusieurs collectifs pour les Kurdes en Pays de la Loire. En janvier 2017, un nouveau collectif, collectif Azadi Kurdistan, est créé en Vendée avec le soutien du $\mathrm{CCK}^{84}$.

Le nombre peu élevé de Kurdes témoigne d'une invisibilité kurde créée par la classification administrative. D'autres dépouillements au sein des archives départementales en Bretagne et Pays de la Loire sont en cours. Le croisement des différentes bases de données, la construction progressive d'arbres généalogiques permettront de retrouver des Kurdes qui n'ont pas fait de demande d'asile et qui ont été catégorisés en tant que Turcs. Dans cette perspective, les témoignages oraux viendront éclairer les comportements, prises de décision, rapports à l'exil et à l'identité, possibilités et choix de réseaux des Kurdes de Turquie dans l'Ouest de la France.

\section{RÉSUMÉ}

Cet article analyse les parcours migratoires de demandeurs d'asile kurdes partis de Turquie pour la France après le coup d'état de 1980. Ces femmes et hommes kurdes se sont installés, après plusieurs déplacements, en LoireAtlantique, département appartenant à un Ouest considéré comme une région de "faible immigration ". Leurs parcours d'installation apparaissent progressivement reliés à ceux de migrants économiques turcs et/ou d'origine kurde arrivés dans la décennie 70. Dès lors, un questionnement s'impose sur les liens existants entre les premières générations de migrants et les réfugiés kurdes. L'exposé de ces deux contextes migratoires permet une réflexion sur les stratégies migratoires mises en œuvre par les Kurdes. Il s'agira de saisir, après leur installation, les liens entre territoire et identité et de comprendre comment ces Kurdes expriment ou dissimulent leur identité en LoireAtlantique.

\section{ABSTRACT}

The present article will analyse the migration journeys of asylum-seeking Kurds who left Turkey for France after the 1980 coup. After several changes and temporary camps, these women and men finally settled in Loire-Atlantique, a department of western France generally considered as a "low immigration area". Their journeys and migration patterns will gradually show growing correlation and links with those of former economic migrants from Turkey in the 1970s, whether Turks or Turkeybased Kurds. This observation then triggers further questioning and investigations related to the relations between these first generations of migrants and the more recent Kurd refugees. Building on the analysis of these two migration backgrounds, this study examines the various migration strategies developed by the Kurds. As they settled in this area, we will look at the identification of links between territory and identity, and understanding if, how, and why, these Kurds tended to express - or hide - their identity in Loire-Atlantique.

84. [http://www.akb.bzh/spip.php?article1140] consulté le 20 janvier 2016. 